\title{
A non-coding RNA locus mediates environment-conditioned male sterility in rice
}

\author{
Danmeng Zhu ${ }^{1}$, Xing Wang Deng ${ }^{1,2}$ \\ ${ }^{1}$ Peking-Yale Joint Research Center for Plant Molecular Genetics and Agrobiotechnology, State Key Laboratory of Protein and \\ Plant Gene Research, College of Life Sciences, Peking University, Beijing 100871, China; ${ }^{2}$ Department of Molecular, Cellular and \\ Developmental Biology, Yale University, New Haven, Connecticut 06520, USA \\ Cell Research (2012) 22:791-792. doi:10.1038/cr.2012.43; published online 20 March 2012
}

The discovery of Nongken 58S, a natural recessive photoperiodsensitive male sterile line, served as a starting point for development of two-line hybrid rice in China. While original Nongken $58 \mathrm{~S}$ in its japonica background is photoperiod-sensitive male sterile, after introgressing of the related locus into indica rice background the same trait become mainly temperature-sensitive male sterile. Although those photoperiod- and temperature-sensitive male sterile lines have been successfully used in two-line hybrid rice seed production, the genetic cause remained a mystery till the two recent publications, with one in Cell Research.

The two-line hybrid rice system, which takes advantage of photoperiodand thermo-sensitive male sterility, has been successfully used in hybrid rice production since 1995 [1]. The story started with a rice variety, Nongken 58, a typical japonica rice, was first introduced in South China in 1957. Because of its high quality and high yield, soon after it became a highly planted cultivar in South China. In 1973, Nongken 58S was found by Mr Mingsong Shi as a

Correspondence: Xing Wang Deng Tel: +1-203-432-8908

E-mail: xingwang.deng@yale.edu spontaneous photoperiod-sensitive genic male sterility (PGMS) mutant, which was quickly applied for two-line hybrid rice development that eventually succeeded in 1995 [2-3]. But the genetic and molecular basis of the PSMS in Nongken 58S is not known. Another mystery in this two-line hybrid breeding is the fact that when the genic male sterile trait is bred into indica rice background, such as Peiai 64S, it become predominantly a temperature-sensitive genic male sterility (TGMS) trait, rather than PGMS. Two recent publications, one in Proc Natl Acad Sci USA from Dr Qifa Zhang lab [4] and one in Cell Res by Dr Yaoguang Liu and Dr Chuxiong Zhuang labs [5], have independently revealed the genetic mutation in the original male sterility trait as a single base C-to-G nucleotide substitution at position 22258571 of chromosome 12 (Figure 1).

Both group claimed that this single nucleotide substitution took place within a non-coding RNA (ncRNA) locus, but suggested distinct non-coding RNA molecules that might be the functional form for this locus. The data from Zhang lab indicated that the presence of abundant level of the 1 236-nt ncRNA (designated LDMAR) is responsible for the male sterility under long-day condition. While Liu and Zhuang labs narrowed down the functional form to a 21-nt small RNA (osa-smR5864w for wild type and osa-smR5864m for the mutant), which is preferentially expressed in young panicles. Liu and Zhuang labs reported that this 21-nt small RNA was actually the product of a 136-nt intermediate precursor by RACE analyses. These two papers together imply a possibility that the 1236 -nt ncRNA could be the primary transcript, which may be processed first into the 136-nt intermediate precursor, then finally into the $21 \mathrm{nt}$ small RNA.

Liu and Zhuang labs also clearly demonstrated that the same C-to-G mutation is responsible for the temperaturesensitive mutation in indica male sterile line Peiai 64S. Thus the same mutant non-coding RNA is responsible for the temperature-sensitive male sterility in the indica background, suggesting that it is the genetic background, but not the ncRNA locus per se, that is responsible for the photoperiod to temperature sensitive male sterility change.

If the 21-nt small RNA is the functional form of this male sterility locus, it will be of great interest to define what are the target genes of this 21-nt small RNA. Further analysis of the expression patterns of those target genes and how they are regulated by photoperiod and temperature as well as the male sterility locus-produced small RNAs (osasmR5864w and osa-smR5864m) should 


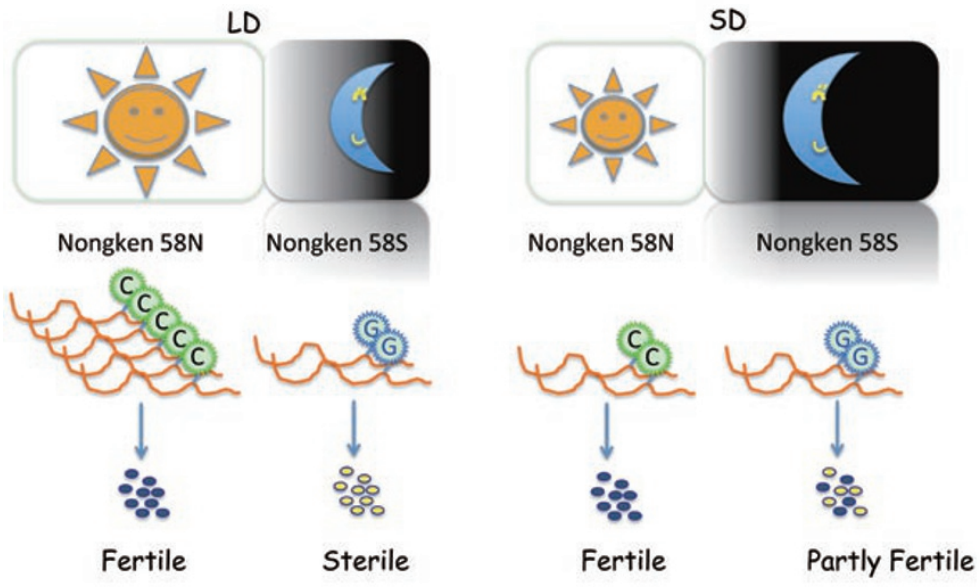

Figure $1 \mathrm{~A}$ ncRNA locus controls photoperiod-sensitive male fertility. A substitution of $C$ to $G$ in chromosome 12 of Nongken $58 \mathrm{~S}$ results in the reduction of the transcription of a small non-coding RNA, which is possibly processed from long precursors, thus causing the long-day-induced male sterility but mostly fertile in short-day condition.

finally reveal the exact molecular basis of the photoperiod and temperaturesensitive male sterility. However, the data presented by both labs have not excluded the possibility that either the 1 236-nt or 136-nt small RNA species could also be a functional form.

Variety of ncRNAs, which function directly as RNA molecules, become one layer of regulators of gene expression in multiple significant biological events [6-7]. Moreover, many structured ncRNAs that express the primary transcript precursors for miRNA- or siRNA-like small RNAs in humans, worms, flies, and plants [8-9]. Case studies supported the general notion that the biogenesis of those small RNAs derived from long precursors are usually dependent on Dicer/Dicer-like proteins or other endonucleases in vivo. Thus, the processing dependency of osa-smR $5846 \mathrm{w} / \mathrm{m}$ should be examined, which will provide valuable information on ncRNA biogenesis. Furthermore, the fact that the SNP with the C-to-G substitution in osa-smR $5864 \mathrm{~m}$ causes reduction of the abundance itself in rice is quite similar to the report that single nucleotide substitution in miR164 affects its accumulation in natural variations [10], which results in the diversity of organ architectures in Arabidopsis. In this case, further investigations will have no doubt to help reveal new insights into small RNA processing and decay in planta.

Small RNAs derived from long precursors are reported to have Argonaute complex loading preference, which potentially linked the small RNA with its mode of function. In addition, Zhang lab provided an interesting observation that the DNA methylation pattern was different in Nongken 58N and Nongken $58 \mathrm{~S}$ regardless of growth stages, with high level of methylation in promoter region but low methylation level within coding region in Nongken 58S [4]. Thus, it will be interesting to know the relationship between small RNA osa-smR5864 and RNAi pathway core component Argonautes, and whether C-to-G substitution in this small RNA leads to the changes of DNA methylation pattern in cis. These studies may shed light on determination of the way of small RNA action in vivo and the mechanistic connection to the PGMS and TGMS traits.

Together, the data from these two reports have defined the genetic cause for a photoperiod- and temperaturesensitive male sterility that all widely used in two-line hybrid breeding. They thus provide a starting point for figuring out how this fascinating ncRNA locus is responsible for the male fertility regulation.

\section{References}

1 Mei M, Chen L, Zhang Z, Li Z, Xu C, Zhang Q. pms 3 is the locus causing the original photoperiod-sensitive male sterility mutation of 'Nongken 58S'. Sci China C Life Sci 1999; 42:316322.

2 Shi Y, Zhao S, Yao J. Premature tapetum degeneration: a major cause of abortive pollen development in photoperiod sensitive genic male sterility in rice. J Integr Plant Biol 2009; 51:774781.

3 Lu Q, Li XH, Guo D, Xu CG, Zhang Q. Localization of $p m s 3$, a gene for photoperiod-sensitive genic male sterility, to a 28.4-kb DNA fragment. Mol Genet Genomics 2005; 273:507-511.

4 Ding J, Lu Q, Ouyang Y, et al. A long noncoding RNA regulates photoperiod-sensitive male sterility, an essential component of hybrid rice. Proc Natl Acad Sci USA 2012; 109:2654-2659.

5 Zhou H, Liu Q, Li J, et al. Photoperiodand thermo-sensitive genic male sterility in rice are caused by a point mutation in a novel noncoding RNA that produces a small RNA. Cell Res 2012; 22:649-660.

6 Taft RJ, Pang KC, Mercer TR, Dinger M, Mattick JS. Non-coding RNAs: regulators of disease. J Pathol 2010; 220:126-139.

7 Mercer TR, Dinger ME, Mattick JS. Long non-coding RNAs: insights into functions. Nat Rev Genet 2009; 10:155159.

8 Ender C, Krek A, Friedlander MR, et $a l$. A human snoRNA with microRNAlike functions. Mol Cell 2008; 32:519528.

9 Taft RJ, Glazov EA, Lassmann T, Hayashizaki Y, Carninci P, Mattick JS. Small RNAs derived from snoRNAs. RNA 2009; 15:1233-1240.

10 Todesco M, Balasubramanian S, Cao $\mathrm{J}$, et al. Natural variation in biogenesis efficiency of individual Arabidopsis thaliana microRNAs. Curr Biol 2012; 22:166-170. 